\title{
Concentrations of glutamate+glutamine are increased in the fetal plasma of pregnant rats fed a diet reflecting that of the poorest socioeconomic groups in Scotland
}

\author{
H. Dasgin, S.M. Hay, S.E. Anderson and W.D. Rees \\ Rowett Institute, University of Aberdeen, Foresterhill Aberdeen, AB25 2ZD, Scotland UK.
}

Pregnant women in deprived populations in Scotland have a poor micronutrient status in addition to a very high intake of saturated fat, refined carbohydrates and salt ${ }^{(1)}$. To investigate the cumulative effects of over-provision of energy substrates, combined with multiple micronutrient deficiencies, we have developed a rodent diet which models the pattern of nutrient intake of those women. One consequence of this diet may be changes in the supply of amino acids to the fetus.

A rodent diet (SIMD) was formulated with a macronutrient composition based on the median intakes of pregnant women in the tenth decile of the Scottish index of multiple deprivation ${ }^{(1)}$. The micronutrient composition of this diet was adjusted using the principle of energy balance. A second diet (SACN) was formulated to incorporate the recommendations of the Scientific Advisory Committee on Nutrition, i.e. low in saturated fat and sugar with reduced salt and with the micronutrients adjusted to meet the recommended intakes. The experimental diets (SIMD and SACN) contained $16.4 \%$ protein by weight; $40 \%$ of the total protein as wheat gluten and $60 \%$ as casein to reflect the human diet. Two groups of nine nulliparous Hooded Lister rats were maintained on either SIMD or SACN diet with a third group fed the widely used AIN-93 G rodent diet as a reference $(20 \%$ protein by weight and $100 \%$ of the total protein as casein). After a 3-week adaptation period, animals were mated and maintained on the same diets until they were killed on day 21 of gestation. A mixture of ${ }^{13} \mathrm{C}$ labelled amino acids was added to samples of maternal and fetal plasma as an internal standard. Amino acids were isolated using ion-exchange chromatography, derivatised with N-tertButyldimethylsilyl-N-methyltrifluoroacetamide (MTBSTFA) and the concentrations determined by GC-MS using isotope dilution.

Maternal plasma amino acid concentrations were very similar in the SIMD and SACN diet groups. Relative to the AIN-93 G reference group, the maternal plasma of the SIMD and SACN groups had lower $(\mathrm{p}<0.05$ to 0.001$)$ concentrations of arginine (average $17 \% \downarrow)$, cysteine $(35 \% \downarrow)$, histidine $(22 \% \downarrow)$, lysine $(33 \% \downarrow)$, valine $(30 \% \downarrow)$ and threonine $(60 \% \downarrow)$.

The fetal amino acid profile reflected that of the dam $\left(\mathrm{R}^{2}>0.94\right)$. Glutamate + glutamine values were increased by $20 \%$ in the SIMD compared with both SACN and AIN-93 G groups $(p=0.029)$. Relative to the AIN-93 G reference group, the fetal plasma of the SIMD and SACN groups had lower $(\mathrm{p}<0.05$ to 0.001$)$ concentrations of cysteine (average $23 \% \downarrow)$, lysine $(19 \% \downarrow)$, glycine $(15 \% \downarrow)$ and threonine $(55 \% \downarrow)$, and higher $(\mathrm{p}<0.05)$ concentrations of methionine $(14 \% \uparrow)$ and phenylalanine $(33 \% \uparrow)$.

In conclusion, a pattern of proteins similar to that found in human diets has modified glutamate+glutamine content in the SIMD fetal plasma. Changes in glutamate and glutamine cycling may influence the growth and development of fetal cells ${ }^{(2)}$. Differences in the concentrations of a number of key amino acids, particularly threonine may be due to the quantity and qualities of proteins in the diets.

This study was supported by The Scottish Government Strategic Research Programme. HD is the recipient of a grant from Newton-Katip Çelebi Fund of The Scientific and Technological Research Council of Turkey (TÜBITAK)-British Council Cooperation.

1. Haggarty P, Campbell DM, Duthie S et al. Piyathilake C, et al. (2009) Br J Nutr 102(10), 1487-97.

2. Wu X, Xie C, Zhang Y et al. (2015) Amino Acids 47(1), 45-53. 